\title{
ON SOME BOUNDS FOR THE MODULI OF ZEROS OF POLYNOMIALS
}

\author{
SANJEEV KUMAR AND HARPREET SINGH MAAN
}

\begin{abstract}
In this article, we specify some sufficient conditions which yield some significant bounds for the moduli of zeros of polynomials with real or complex coefficients. The paper proves the main result of [M. H. Gulzar, S. Bashir, Bounds for the moduli of zeros of polynomials, International Research Journal of Advanced Engineering and Science, 2:2 (2017), 51-53] as a special case.
\end{abstract}

\section{Introduction}

In 2004, Shah et al. proved that if $f(z)=\sum_{i=0}^{n} a_{i} z^{i}$ be a polynomial with real or complex coefficients such that $\sum_{i=0}^{n-1}\left|a_{i}\right| \leq\left|a_{n}\right|$, then the zeros of $f(z)$ lie in the region $|z| \leq 1$ (see [4], [2, Theorem D]). Recently, Gulzar et al. with a weaker hypothesis proved the following results ([2, Theorem 1, Theorem 2]).

Theorem 1.A. Let $f(z)=\sum_{i=0}^{n} a_{i} z^{i}$ be a polynomial of degree $n$ with real or complex coefficients satisfying $\sum_{i=0}^{n}\left|a_{i}-a_{i-1}\right| \leq\left|a_{n}\right|, a_{-1}=0$, then the zeros of $f(z)$ lie in the annulus $\frac{\left|a_{0}\right|}{2\left|a_{n}\right|-\left|a_{0}\right|} \leq$ $|z| \leq 1$.

Theorem 1.B. Let $f(z)=\sum_{i=0}^{n} a_{i} z^{i}$ be a polynomial of degree $n$ with real or complex coefficients satisfying $\sum_{i=0}^{n}\left|a_{i}-a_{i-1}\right| \leq\left|a_{n}\right|, a_{-1}=0$, then the zeros of $f(z)$ lie in the annulus

$$
\frac{\left|a_{0}\right|}{\left|a_{n}\right| r^{n}(r+1)-\left|a_{0}\right|} \leq|z| \leq 1, \text { if } r \geq 1
$$

and

$$
\frac{\left|a_{0}\right|}{\left|a_{n}\right| r\left(r^{n}+1\right)-\left|a_{0}\right|} \leq|z| \leq 1, \text { if } r \leq 1 \text {. }
$$

Received January 24, 2018, accepted July 16, 2018.

2010 Mathematics Subject Classification. Primary: 30C10, Secondary: 30C15.

Key words and phrases. Polynomials, zeros of polynomials.

Corresponding author: Sanjeev Kumar. 
In this article, we aim at devising some suffiicient conditions which yield some succint bounds for the moduli of zeros of polynomials. It must be pointed out that Theorems 1.A and 1.B, mentioned above are infact special cases of the results proved here, which are precisely stated below.

Theorem 1.1. Let $f(z)=\sum_{i=0}^{n} a_{i} z^{i}$ be a polynomial of degree $n$ with real or complex coefficients such that for any real number $\alpha \geq 1, \sum_{i=0}^{n}\left|a_{i}-\alpha a_{i-1}\right| \alpha^{n-i} \leq\left|a_{n}\right|, a_{-1}=0$. Then the zeros of $f(z)$ lie in the annulus $\frac{\left|a_{0}\right|}{\left|a_{n}\right| r^{n}(\alpha r+1)-\left|a_{0}\right|} \leq|z| \leq \frac{1}{\alpha}$ for $r \geq 1$.

Theorem 1.2. Let $f(z)=\sum_{i=0}^{n} a_{i} z^{i}$ be a polynomial of degree $n$ with real or complex coefficients such that for any real number $\alpha \geq 1, \sum_{i=0}^{n}\left|a_{i}-\alpha a_{i-1}\right| \alpha^{n-i} \leq\left|a_{n}\right|, a_{-1}=0$. Then the zeros of $f(z)$ lie in the annulus $\frac{\left|a_{0}\right|}{\left|a_{n}\right| r\left(\alpha r^{n}+1\right)-\left|a_{0}\right|} \leq|z| \leq \frac{1}{\alpha}$ for $r \leq 1$.

Corollary 1.3. Let $f(z)=\sum_{i=0}^{n} a_{i} z^{i}$ be a polynomial of degree $n$ with real or complex coefficients such that $\sum_{i=0}^{n}\left|a_{i}-2 a_{i-1}\right| 2^{n-i} \leq\left|a_{n}\right|, a_{-1}=0$. Then the zeros of $f(z)$ lie in the annulus $\frac{\left|a_{0}\right|}{3\left|a_{n}\right|-\left|a_{0}\right|} \leq|z| \leq \frac{1}{2}$.

Corollary 1.4. Let $f(z)=\sum_{i=0}^{n} a_{i} z^{i}$ be a polynomial of degree $n \geq 2$ with real or complex coefficients such that

$$
\sum_{i=0}^{n}\left|a_{i}-2 a_{i-1}\right| 2^{n-i} \leq 2^{n}, a_{0}=1, a_{-1}=0 .
$$

Then the zeros of $f(z)$ lie in the annulus $\frac{1}{2^{n-1}} \leq|z| \leq \frac{1}{2}$.

Example 1.5. The zeros of the polynomial $f(z)=\sum_{i=0}^{n} z^{i}$ lie on the unit circle.

Remark. Theorems 1.1 and 1.2 together on taking $\alpha=1$ yields Theorem 1.B. Theorem 1.A can be obtained from either Theorem 1.1 or Theorem 1.2 on taking $\alpha=1$ and $r=1$. Theorems 1.1 and 1.2 coincide for $\alpha=1$ and $r=1$. Corollary 1.3 follows immediately from Theorem 1.1 or Theorem 1.2 with $\alpha=2$ and $r=1$. Corollary 1.4 follows from Theorem 1.2 on taking $\alpha=2, r=\frac{1}{2},\left|a_{0}\right|=1$ and $\left|a_{n}\right|=2^{n}$. Example 1.5 can be quickly deduced from either Theorem 1.1 or Theorem 1.2 with $\alpha=1=r$. 
We now proceed to the next section containing some results which pave the way for the construction of the proof of Theorem 1.1 and Theorem 1.2.

\section{Preliminary results}

The following auxiliary results are necessary for establishing Theorem 1.1 and Theorem 1.2 .

Lemma 2.1. Let $f(z)=\sum_{i=0}^{n} a_{i} z^{i}$ be a polynomial of degree $n$ with real or complex coefficients such that for any real number $\alpha \geq 1$,

$$
\sum_{i=0}^{n}\left|a_{i}-\alpha a_{i-1}\right| \alpha^{n-i} \leq\left|a_{n}\right|, a_{-1}=0 .
$$

Then the zeros of $f(z)$ lie in $|z| \leq \frac{1}{\alpha}$.

Proof. Consider the polynomial

$$
g(z)=(1-\alpha z) f(z)=-a_{n} z^{n+1}+\sum_{i=0}^{n}\left(a_{i}-\alpha a_{i-1}\right) z^{i} .
$$

If $|z|>\frac{1}{\alpha}$, then $\alpha|z|>1$ and we observe that the absolute value of $g(z)$ equals

$$
\left|-a_{n} \alpha z^{n+1}+\sum_{i=0}^{n}\left(a_{i}-\alpha a_{i-1}\right) z^{i}\right| \geq\left|-a_{n} \alpha z^{n+1}\right|-\left[\sum_{i=0}^{n}\left|a_{i}-\alpha a_{i-1}\right|\left|z^{i}\right|\right] .
$$

Further observe that

$$
\left|-a_{n} \alpha z^{n+1}\right|-\left[\sum_{i=0}^{n}\left|a_{i}-\alpha a_{i-1}\right||z|^{i}\right]=\left|z^{n}\right|\left\{\left|a_{n} \alpha\right||z|-\left[\sum_{i=0}^{n} \frac{\left|a_{i}-\alpha a_{i-1}\right|}{|z|^{n-i}}\right]\right\} .
$$

Keeping in mind that $|z|>\frac{1}{\alpha}$, we get that the last inequality is strictly greater than

$$
\left|z^{n}\right|\left\{\left|a_{n} \alpha\right||z|-\left[\sum_{i=0}^{n}\left|a_{i}-\alpha a_{i-1}\right| \alpha^{n-i}\right]\right\}
$$

which is infact atleast

$$
\left|z^{n}\right|\left(\left|a_{n} \alpha\right||z|-\left|a_{n}\right|\right)=\left|z^{n}\right|\left|a_{n}\right|(|\alpha||z|-1)>0 .
$$

Thus the polynomial $g(z)$ has no zeros in the region $|z|>\frac{1}{\alpha}$. Therefore all the zeros of $g(z)$ and hence of $f(z)$ lie in $|z| \leq \frac{1}{\alpha}$.

The following result is the celebrated Schwarz Lemma ([1, \$1, Theorem, p. 260], [3, Chapter VII, $\$ 1$, Theorem 1.1, p. 210]), which is a simple yet powerful tool in establishing bounds on the moduli of zeros of polynomials with real or complex coefficients. 
Lemma 2.2. Let $f(z)$ be analytic for $|z|<1$. Suppose that $|f(z)| \leq 1$ for all $|z|<1$ and $f(0)=0$. Then $|f(z)| \leq|z|$ for $|z|<1$. Further, if for some point $z_{0} \neq 0,\left|f\left(z_{0}\right)\right|=\left|z_{0}\right|$, then $f(z)=\lambda z$ for some constant $\lambda$ of unit modulus.

With the above results, we proceed to the next section to prove the main results of this article.

\section{Proof of Theorems 1.1 and 1.2 .}

Lemma 2.1 paves the way for the assertion that the zeros of polynomials satisfying the hypothesis of Theorems 1.1 and 1.2 lie in the region $|z| \leq \frac{1}{\alpha}$. The proofs of the inequality associated with the inner boundary of the annuli in Theorems 1.1, 1.2 runs on the same lines as that of Theorem 2 of [2].

Proof of Theorem 1.1. With $f(z), g(z)$ as in the proof of Lemma 2.1, consider the polynomial $h(z)$ such that $g(z)-h(z)=a_{0}$ so that

$$
h(z)=-a_{n} \alpha z^{n+1}+\sum_{i=1}^{n}\left(a_{i}-\alpha a_{i-1}\right) z^{i} .
$$

Now that $h(z)$ is analytic for $|z| \leq r, h(0)=0, r \geq 1$, observe that

$$
\begin{aligned}
|h(z)| & =\left|-a_{n} \alpha z^{n+1}+\sum_{i=1}^{n}\left(a_{i}-\alpha a_{i-1}\right) z^{i}\right| \\
& \leq\left|a_{n} \alpha\right| r^{n+1}+r^{n}\left[\sum_{i=0}^{n}\left|a_{i}-\alpha a_{i-1}\right|\right]-\left|a_{0}\right| \\
& \leq\left|a_{n} \alpha\right| r^{n+1}+r^{n}\left[\sum_{i=0}^{n}\left|a_{i}-\alpha a_{i-1}\right| \alpha^{n-i}\right]-\left|a_{0}\right|,
\end{aligned}
$$

which in view of the hypothesis of Theorem 1.1 yields that the last expression is less than or equal to

$$
\left|a_{n} \alpha\right| r^{n+1}+r^{n}\left|a_{n}\right|-\left|a_{0}\right|=\left|a_{n}\right| r^{n}(\alpha r+1)-\left|a_{0}\right| .
$$

Now by Lemma 2.2, for $r \geq 1$ and $|z| \leq r$, we have

$$
|h(z)| \leq\left[\left|a_{n}\right| r^{n}(\alpha r+1)-\left|a_{0}\right|\right]|z|
$$

and hence $g(z) \geq\left|a_{0}\right|-|h(z)| \geq\left|a_{0}\right|-\left[\left|a_{n}\right| r^{n}(\alpha r+1)-\left|a_{0}\right|\right]|z|$, which is positive iff $|z|<\frac{\left|a_{0}\right|}{\left|a_{n}\right| r^{n}(\alpha r+1)-\left|a_{0}\right|}$. Therefore no zeros of $g(z)$ and hence $f(z)$ lie in the region $|z|<\frac{\left|a_{0}\right|}{\left|a_{n}\right| r^{n}(\alpha r+1)-\left|a_{0}\right|}$ for $r \geq 1$. Thus all the zeros of $f(z)$ lie in the annulus $\frac{\left|a_{0}\right|}{\left|a_{n}\right| r^{n}(\alpha r+1)-\left|a_{0}\right|} \leq|z| \leq \frac{1}{\alpha}$. This proves the theorem. 
Proof of Theorem 1.2. With $f(z), g(z)$ and $h(z)$ as considered in the proof of Theorem 1.1, note that for $r \leq 1$ and $|z| \leq r$,

$$
\begin{aligned}
|h(z)| & =\left|-a_{n} \alpha z^{n+1}+\sum_{i=1}^{n}\left(a_{i}-\alpha a_{i-1}\right) z^{i}\right| \\
& \leq\left|a_{n} \alpha\right| r^{n+1}+r\left[\sum_{i=0}^{n}\left|a_{i}-\alpha a_{i-1}\right|\right]-\left|a_{0}\right| \\
& \leq\left|a_{n} \alpha\right| r^{n+1}+r\left[\sum_{i=0}^{n}\left|a_{i}-\alpha a_{i-1}\right| \alpha^{n-i}\right]-\left|a_{0}\right|,
\end{aligned}
$$

which in view of the hypothesis of Theorem 1.2 leads to the fact that the last expression is less than or equal to

$$
\left|a_{n} \alpha\right| r^{n+1}+r\left|a_{n}\right|-\left|a_{0}\right|=\left|a_{n}\right| r\left(\alpha r^{n}+1\right)-\left|a_{0}\right| .
$$

Now by virtue of Lemma 2.2, for $r \leq 1$ and $|z| \leq r$, we have

$$
|h(z)| \leq\left[\left|a_{n}\right| r\left(\alpha r^{n}+1\right)-\left|a_{0}\right|\right]|z|
$$

and hence $g(z) \geq\left|a_{0}\right|-|h(z)| \geq\left|a_{0}\right|-\left[\left|a_{n}\right| r\left(\alpha r^{n}+1\right)-\left|a_{0}\right|\right]|z|$, which is positive iff $|z|<\frac{\left|a_{0}\right|}{\left|a_{n}\right| r\left(\alpha r^{n}+1\right)-\left|a_{0}\right|}$. Therefore no zeros of $g(z)$ and hence $f(z)$ lie in the region $|z|<\frac{\left|a_{0}\right|}{\left|a_{n}\right| r\left(\alpha r^{n}+1\right)-\left|a_{0}\right|}$ for $r \leq 1$. Thus all the zeros of $f(z)$ lie in the annulus $\frac{\left|a_{0}\right|}{\left|a_{n}\right| r\left(\alpha r^{n}+1\right)-\left|a_{0}\right|} \leq|z| \leq \frac{1}{\alpha}$ and the theorem follows.

Remark. In Theorems 1.1 and 1.2, in case $\left|a_{0}\right|=\left|a_{n}\right|=1, r=1$, then the roots of the polynomial $f(z)$ lie on the circle $|z|=\frac{1}{\alpha}$. Hence in particular with $\alpha=1$, the zeros of the polynomial $f(z)=\sum_{i=0}^{n} z^{i}$, lie on the unit circle.

\section{References}

[1] T. W. Gamelin, Complex Analysis, Springer-Verlag, New York, 2001.

[2] M. H. Gulzar and S. Bashir, Bounds for the moduli of zeros of polynomials, IRJAES, 2:2 (2017), 51-53.

[3] S. Lang, Complex Analysis, $4^{t h}$-Edition, Springer Science+Business Media, Inc., New York, 1999.

[4] W. M. Shah and A. Liman, On bounds for the zeros of polynomials, Anal. Theory Appl., 20:1 (2004), $16-27$.

Department of Mathematics, SGGS College, Sector-26, Chandigarh-160019, India.

E-mail: sanjeev_kumar_19@yahoo.co.in

Department of Mathematics, SGGS College, Sector-26, Chandigarh-160019, India.

E-mail: hpsmaan@gmail.com 